\title{
Evolutionary insights from the Vikings
}

primordial
eukaryotic
vesicular and
trafficking
components
are derived
from our
archaeal
ancestor

The emergence of the eukaryotic cell during evolution gave rise to all complex life forms on Earth, including multicellular organisms such as animals, plants and fungi. However, the origin of eukaryotes and their characteristic structural complexity has remained a mystery. The most recent insights into eukaryogenesis support the endosymbiotic theory, which proposes that the first eukaryotic cell arose from archaea through the acquisition of an alphaproteobacterial endosymbiont. Using phylogenomic analyses of uncultivated archaeal lineages, Zaremba-Niedzwiedzka et al. provide biological insights into the archaeal ancestor of eukaryotes and the primal stages of eukaryogenesis.

First, the authors identified and characterized phyla that are closely related to the archaeal phylum Lokiarchaeota. Previous studies had placed the Lokiarchaeota as the most closely related archaea to eukaryotes as they have genes that are typically associated with eukaryotic functions, such as membrane remodelling. The authors identified two additional related phyla that they grouped into a superphylum and named

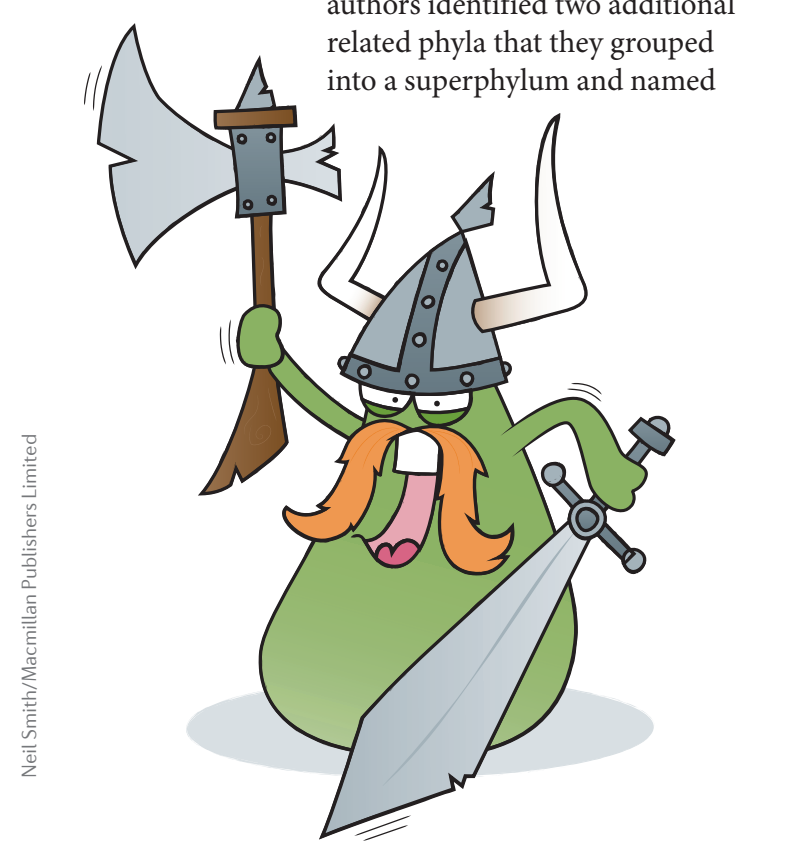

ASGARD - after the invisible 'Gods of Asgard' in Norse mythology. The superphylum consists of the previously identified Lokiarchaeota and Thorarchaeota phyla, and the newly identified Odinarchaeota and Heimdallarchaeota phyla. Using phylogenomics, they discovered a strong phylogenetic association between ASGARD lineages and eukaryotes that placed the eukaryote lineage in close proximity to the ASGARD superphylum.

Next, to investigate the emergence of eukaryotic gene content in the ASGARD lineages, the authors searched for the presence of proteins that are unique to eukaryotes (eukaryotic signature proteins) and found that ASGARD lineages were enriched for these proteins. For example, homologues of eukaryotic endosomal sorting complex required for transport (ESCRT) systems, ubiquitylation proteins and orthologues of essential eukaryotic cytoskeletal components were enriched. Indeed, several key eukaryotic cytoskeletal proteins including tubulin, gelsolin-domain and profilin-domain proteins, and homologues of the actin-related protein 2/3 (ARP2/3) complex were identified, which indicates that members of the ASGARD superphylum have several fundamental building blocks that are required for the evolution of a sophisticated eukaryotic-like cytoskeleton. Interestingly, the authors also found that genes that encode ESCRT and ubiquitin modifier system proteins were co-organized in the same gene clusters, which indicates that ESCRT-mediated protein degradation might operate in members of the ASGARD superphylum, a system that was only thought to exist in eukaryotes. Similarly, an epsilon DNA polymerase subunit was identified in the Heimdallarchaeota that represents the closest known homologue of eukaryotic epsilon DNA polymerases identified thus far.

Members of the ASGARD superphylum were particularly enriched for eukaryotic signature proteins that are involved in intracellular trafficking and secretion. Several proteins contained domain signatures of eukaryotic transport protein particle (TRAPP) complexes, which are involved in transport from the endoplasmic reticulum through the Golgi apparatus in eukaryotes. In addition, eukaryotic homologues of the Sec23/24 family that are components of the COPII vesicle coatomer protein complex were found in the Thorarchaeota, which prompted the authors to search for further eukaryotic coatomer proteins. Eukaryotic coatomer proteins are composed of amino-terminal $\beta$-propeller folds followed by a carboxy-terminal a-solenoid fold; both of these folded domains are extremely rare in prokaryotic proteins. Remarkably, the authors identified further eukaryotic-like coatomer proteins in the ASGARD superphylum, including proteins that contain both $\beta$-propeller and $\alpha$-solenoid folds, which indicates that primordial eukaryotic vesicular and trafficking components are derived from our archaeal ancestor.

In sum, this study provides an exciting new perspective on the origin of eukaryotic membrane trafficking, eukaryogenesis and the evolution of a compartmentalized and structurally complex eukaryotic cell, which ultimately led to the acquisition of new cellular functions that drove the evolution of multicellular life on Earth.

Ashley York

ORIGINAL ARTICLE Zaremba-Niedzwiedzka, K. et al. ASGARD archaea illuminate the origin of eukaryotic cellular complexity. Nature http://dx. doi.org/10.1038/nature21031 (2017) 